\title{
ESTUDO DE CASO DE UMA PEQUENA CLÍNICA: FINANÇAS E MARKETING
}

CASE STUDY OF A SMALL CLINIC : FINANCE AND MARKETING

Djair Picchiai', Isabella Ulson Resky²

RECEBIDO: 10/12/2015 | 09/05/2016

DOI: $10.5902 / 2317175820604$

\section{RESUMO}

O objetivo deste artigo foi analisar as práticas de gestão utilizadas pelos gerentes e pelo proprietário de uma pequena empresa (PE) prestadora de serviços especializados em oftalmologia, denominada HC Jundiaí. Caracterizando-se como um estudo de caso qualitativo, descritivo e exploratório (YIN, 2001; VERGARA, 2007). Analisaram-se as áreas funcionais; finanças e marketing/comercialização como fatores constitutivos das estratégias utilizadas. Focou-se nas áreas de marketing e finanças que se constituem em áreas core das micro e pequenas empresas (MPE's). A metodologia constituiu-se de entrevistas estruturadas junto com gestores e proprietário, o preenchimento de um questionário contendo 50 perguntas, coleta de dados da empresa, consulta a drevistaocumentos e a observação em campo dos por nove meses. A intuição, a vivência e a experiência do proprietário, foram consideradas relevantes no processo de tomada de decisão. O contexto das MPE's tem características muito específicas, muitas das ferramentas gerenciais (orçamento, análise de valor, descrição do mercado) não são utilizadas, apesar da complexidade do ambiente organizacional assim o exigir. A sustentabilidade da clínica nos últimos 20 anos aponta para resultados financeiros e comerciais satisfatórios com margens de rentabilidade menores.

Palavras-chave: pequena empresa, marketing, resultados financeiros

1Professor da EAESP-FGV e titular do mestrado profissional da FACCAMP 2Graduada em administração de empresa, pela Eaesp - Fgv 2017. Trabalha na Accenture Brasil. Business Análises 2018. 
ESTUDO DE CASO DE UMA PEQUENA CLÍNICA:

FINANÇAS E MARKETING

\begin{abstract}
The aim of this paper was to analyze management practices used by managers and the owner of a small business (PE) called HC Jundiaí. It constitutes a provider of specialized services in ophthalmology clinic. We conducted an exploratory analysis in depth the operation of the clinic and its management practices. Characterized as a qualitative case study, descriptive and exploratory (YIN, 2001; VERGARA, 2007). They analyzed the functional areas; finance and marketing / marketing as constituent factors of the strategies used. Focused in the areas of marketing and finance which constitute core areas of micro and small enterprises (MSEs). The methodology consisted of structured interviews with managers and owner, plus the completion of a questionnaire containing 50 questions. It also promoted the collection of company data, consultation documents, and observation of the researchers of its operation for nine months. Intuition, the experience and the experience of the owner, were considered relevant in the decision-making process. The theories on management, marketing and finance, explain in part the results and other shares of the company. The context of MPE's has very specific characteristics, many of the management tools (budget, description of the clientele) are not used, despite the complexity of the organizational environment so requires.
\end{abstract}

Keywords: small business, guerrilla marketing, financial results

\title{
1 Introdução
}

As micro e pequenas empresas (MPE's) são preponderantemente empresas familiares e ocupam um espaço importante na economia brasileira (SEBRAE, 2012). Além disso, elas estão presentes nos vários setores e ramos da economia, apresentando alto grau de heterogeneidade entre si (LEONE, 1999). As variações nas estratégias das MPE's ocorrem nas formas e conteúdos de suas atividades core que são finanças e marketing (PICCHIAI, 2012). Estas formas e conteúdos, são frutos das decisões e ações tomadas pelos sócios proprietários fundadores das MPE's.

As MPE's apresentam características de forte adaptação às mudanças, sendo que elas aparecem e desaparecem do mercado com maior velocidade do que as médias e grandes empresas (SEBRAE, 2008). Segundo o Serviço Brasileiro de Apoio às Micro e Pequenas Empresas (SEBRAE, 2012), as MPE's representam aproximadamente $99 \%$ do total de empresas formalmente estabelecidas. O SEBRAE classifica as MPE's pelo número de empregados, independente do seu faturamento.

As MPE's, segundo Cadastro Geral de Empregados e DesempregadosCAGED, são responsáveis, em outubro de 2013 , por $86 \%$ da geração líquida de empregos, enquanto que as médias e grandes empresas respondem por $14 \%$ da geração líquida de empregos, (BRASIL, 2014).

Existem diferentes critérios para definição de MPE's, tais como o do SEBRAE, do BNDES e do Banco Central. Adotou-se o critério do SEBRAE. A literatura aponta dificuldades de se ter um critério único na definição do que é uma MPE (LEONE, 1999). Apesar de adotar o critério de classificação das MPE's 
do SEBRAE (2008), esse estudo de caso não se afastou significativamente dos demais critérios adotados pelo BNDES e Banco Central.

Este artigo foi realizado devido à necessidade e à importância de se conhecer e discutir as práticas de gestão de uma pequena empresa em campo, especializada em conhecimento e com base tecnológica de uma região do Estado de São Paulo, nas áreas de finanças e marketing. Portanto, busca-se analisar as áreas core de uma MPE, que são finanças e marketing/ comercialização.

\section{Referencial teórico}

Como se trata de um projeto que aborda duas áreas para uma clínica especializada em oftalmologia, finanças e marketing, a teoria envolve obras que tratam de assuntos nesses campos. Assim, é possível criar uma base teórica, contendo marketing e finanças sustentando o modelo de análise.

\subsection{Aumento da Tangibilidade de Serviços}

O artigo de David Tarn (2008) busca estudar a intangibilidade que existe na indústria de serviços e suas consequências. Estudos mostram que a intangibilidade pode levar os consumidores a se sentirem em uma posição arriscada, de menor confiança nos provedores do serviço, além de criar expectativas divergentes e dificultar a avaliação do serviço. Assim, aumentamse as chances dos clientes não terem suas expectativas atendidas. Isso, por consequência, pode aumentar o custo variável para atender cada cliente, diminuindo a eficiência e produtividade.

Muitos estudos têm um grande foco no aumento da tangibilidade operacional, enquanto Tarn (2008) destaca que o que ele chama de MarketingBased Tangibilisation (MBT) pode ser uma estratégia diferencial, por focar no aumento da tangibilidade através do marketing. O MBT se mostra eficiente por ajudar a reduzir a divergência entre expectativas de seus consumidores. Além disso, segundo o autor, um MBT bem estruturado ajuda a reduzir as incertezas e riscos percebidos pelo consumidor antes mesmo deste utilizar do serviço. Por último, o MBT, ao diminuir a intangibilidade dos serviços, ajuda o consumidor a formar suas expectativas com menor incerteza e percepção de risco, evitando avaliações fora do quadro de resposta esperado. Isso aumenta a produtividade do serviço.

Acredita-se que este modelo, aplicável às empresas de serviços, ou forma geral, é relevante para os clínicos oftalmológicos que utilizam conhecimentos especializados e tecnológicos.

2.2 Importância dos médicos no marketing hospitalar e das clínicas

O artigo dos autores Bloching, Stock e Scheel (2008), publicado no Journal 
ESTUDO DE CASO DE UMA PEQUENA CLÍNICA:

FINANÇAS E MARKETING

of Medical Market, busca evidenciar, a partir do estudo do mercado hospitalar alemão, como hospitais estão cada vez mais aderidos aos princípios de mercado para conquistar clientes. Em um mercado cada vez mais competitivo, grandes hospitais adotam um marketing que foca cada vez mais no diferencial de seus médicos. Este foco nos médicos é muito relevante para as pequenas clínicas especializadas. $\mathrm{O}$ caso do $\mathrm{HC}$ Jundiaí não foge a essa regra.

Em um cenário cada vez mais competitivo no setor de saúde (mais hospitais, clínicas e centros de diagnóstico), o foco apenas em redução de custos pode ser traiçoeiro, uma vez que a qualidade do serviço oferecido pode ser deteriorada (PORTER; TEISBERG, 2007). A tentativa de redução de custos pode afetar a operacionalidade do serviço, em que pacientes esperam mais para ter um atendimento relativamente pior. O impacto deste novo serviço pode não atender às expectativas dos clientes e diminuir mais ainda as receitas da clínica, fazendo com que este entre em um ciclo vicioso.

Bloching, Stock e Scheel (2008) destacam, também, que a tendência é que os pacientes sejam mais autônomos em suas decisões, buscando sempre mais informações sobre os serviços e demandando mais transparência sobre os métodos de tratamento e possíveis alternativas. Os pacientes estão preocupando e, consequentemente, gastando mais com serviços de saúde, por isso aumentam seu grau de exigência. Em consequência disso, além de mais transparentes, os hospitais devem focar em seus diferenciais. A popularidade e reputação de um médico se torna um fator importante em atrair clientes para o hospital. Médicos de destaque são peças-chave para atingir a satisfação do cliente.

Com base nisso, os autores destacam a importância do marketing hospitalar ser mais transparente. A estratégia de marketing dos hospitais deve focar nos seus diferenciais, ou seja, nos fatores que os distinguem de competidores. Processos médicos, atendimento e equipe de qualidade são os grandes focos do marketing hospitalar. A estratégia deve ser adequada ao ambiente de negócios que se está inserido e para isso é preciso conhecer o público-alvo e suas características, as oportunidades existentes e como ter um modelo de preços que se encaixe nesse cenário. Em consequência disso, o hospital irá focar sua estrutura organizacional e seus investimentos para confirmar diante ao público a qualidade de seu serviço, que deve ser aprimorada cada vez mais. Isso faz com que o hospital mantenha-se competitivo e consiga aumentar sua demanda, mesmo em um cenário de extrema concorrência e pressão por uma melhor estrutura de custos (PORTER; TEISBERG, 2007).

Essa estratégia de marketing é importante e deverá ser utilizada como diferencial pela clínica, destacando a qualidade da sua equipe de médicos. Focar na qualidade de formação de sua equipe de oito médicos (ou dezesseis) e no seu potencial de oferecer um serviço de ótima qualidade deve ser um dos focos visados pela clínica. O proprietário e os gestores têm essa percepção. 
2.3 Marketing de Guerrilha.

A ideia de marketing de guerrilha tem, em sua abordagem, o fato de que os mercados estão cada vez mais competitivos e que o marketing está mais sofisticado. Esse conceito busca mostrar estratégias de como empresas de pequeno e médio porte podem, por meio de ações práticas e competitivas, obter lucros e superar a concorrência.

Para Kotler (2000), o marketing busca identificar e entender as necessidades humanas e sociais. Já na obra de Jay Conrad Levinson (1989), o autor destaca que é necessário as empresas se tornarem guerrilheiras, e para isso elas precisam abrir sua mentalidade, planejar seu ataque de marketing, definir o ataque em si (senão é melhor nem atacar) e precisam, entre outras exigências, saber explicar sobre a empresa para o cliente, de maneira que este ajude na prospecção. Para Levinson (1989), o bom guerrilheiro consegue facilitar suas atividades de marketing por meio da repetição, e boa parte das suas armas de marketing (como funcionários e clientes, por exemplo) não requerem maiores gastos.

Levinson (1989) destaca as cem armas que um guerrilheiro de marketing pode utilizar para superar a concorrência e adquirir longevidade. Seguem as armas mais interessantes para serem incorporadas na estratégia: 1) nome: fácil de pronunciar e passa a imagem de um hospital com competência e foco nos serviços que oferecem, que são voltados para oftalmologia; 2) identidade: transmitir a personalidade da empresa de maneira clara durante o processo de comercialização; 3) estabelecimento de preço: escolher o nicho de preço que melhor atenda o público focado; 4) cartazes externos: a divulgação fora da empresa chama atenção das pessoas e encaminha as mesmas para o estabelecimento; 5) limpeza: a bagunça pode passar uma imagem de desordem e descuido, podendo afastar clientes. Em casos de hospital, a limpeza é um fator essencial; 6) localização: localizar em pontos estratégicos e saber explorar o potencial da região é importante para atingir lucros; 7) follow-up: o acompanhamento após a realização do serviço é a chave para um bom relacionamento com os clientes; 8) sorrisos: importantes para fazer com clientes sintam-se especiais. Funcionários que se mostram alegres geram uma melhor impressão nos clientes; 9 ) tempo de contato com o cliente: o momento que se passa com o cliente é uma oportunidade de marketing. O tempo com o cliente deve ser usado da maneira mais agradável possível, para que assim o relacionamento seja intensificado; 10) divulgação boca-a-boca: é possível aumentar a divulgação oferecendo um serviço excelente, folhetos informativos e um fluxo constante de informações e mala-direta. Estabelecer vínculo com clientes é fundamental; 11) consultas: são como amostras e funcionam muito bem para empresas do setor de serviços. Consultas grátis ajudam na venda de muitos produtos; 12) anúncios em revistas: forma de dar credibilidade para a empresa. É possível utilizar tanto revistas técnicas como revistas de informações 
gerais; 13) outdoor: são vistos por várias pessoas e fazem com que pessoas lembrem de sua empresa. O segredo para o sucesso é: boa localização, texto curto; 14) credibilidade: arma essencial para transformar clientes potenciais em consumidores, pois, com credibilidade, as pessoas acreditarão em suas qualidades, valores e na empresa; 15) clientes satisfeitos: o cliente satisfeito com o serviço e preço é o aliado mais poderoso de marketing (TURRINI,2001). Essas pessoas criam o boca-a-boca voluntariamente.

Vale ressaltar que, apesar de a obra de Levinson (1989) ter sido elaborada bem antes da internet, a ideia do estudo deste referencial é justamente extrair táticas que são fundamentais para que a qualidade do serviço da clínica seja superior aos concorrentes. Apesar da obra em questão ser mais focada para mercados extremamente competitivos, suas estratégias para obtenção de vantagem competitiva são aplicáveis ao cenário e ao tipo de serviço que se oferece.

\subsection{Avaliação de investimento e medidas de viabilidade}

De acordo com a obra Financial Management, de Eugene Brigham e Michael Ehrhardt (2005), existem seis principais medidas para analisar um investimento e sua respectiva viabilidade: Valor Presente Líquido (VPL), Taxa Interna de Retorno (TIR), Taxa Interna de Retorno Modificada (TIRM), Índice de Lucratividade (IL), Payback e Payback Descontado.

O Valor Presente Líquido (VPL) consiste basicamente em uma técnica que vai fazer uma mensuração direta de quanto de valor o projeto irá adicionar ao(s) acionista(s), através do cálculo que envolve a somatória das gerações de caixa do projeto, descontados pela taxa mínima de atratividade (taxa WACC), considerando que o desembolso inicial é a primeira geração de caixa (e, por ser saída de caixa, entra como valor negativo no cálculo). O VPL considera, além disso, que a taxa de reinvestimento em questão é o próprio custo do capital (taxa WACC).

Já a Taxa Interna de Retorno (TIR) e a TIR Modificada (TIRM) têm como objetivo mensurar a lucratividade e expressá-la em percentagem de retorno, sendo que a segunda considera um cenário de reinvestimento mais realístico e recomendado do que a primeira. O Índice de Lucratividade (IL) mede a lucratividade em relação ao total do investimento inicial

Por fim, as medidas de Payback e Payback Descontado vão analisar o investimento do ponto de vista de liquidez e retorno do desembolso inicial, sendo que o segundo desconta as gerações futuras de caixa ao longo do tempo pela taxa mínima de atratividade do negócio (enquanto que o primeiro não desconta esses valores ao longo do tempo). Vale ressaltar que um payback mais longo significa menor liquidez e um maior intervalo de tempo para "recuperar" os gastos iniciais.

Em conclusão, pode-se verificar que existem diferentes maneiras de realizar o cálculo da viabilidade de um investimento ou um projeto, e que cada medida fornece uma diferente visão financeira. Grandes empresas com elaborados softwares financeiros costumam realizar o cálculo de todas as seis métricas. No 
caso da clínica, porém, as métricas utilizadas serão VPL, IRR e Payback, por serem medidas mais elaboradas para verificar geração de valor e liquidez do negócio.

Além disso, outra importância da análise financeira e econômica do hospital é para ajudar a medir sua vantagem competitiva. Primeiro, mede-se a vantagem financeira usando vários índices calculados com os demonstrativos financeiros da empresa, tais como Return on Equity (ROE) e índice preço-lucro (P/L). Já o desempenho econômico compara o nível de retorno de uma empresa com o custo de capital, ou seja, a taxa de retorno requerida por seus investidores.

\section{Método de pesquisa}

Existem duas categorias de pesquisa: a exploratória e a descritiva. A pesquisa exploratória tem como foco a abordagem inicial e descoberta sobre um determinado tópico, detendo, assim, um caráter qualitativo (VERGARA, 2007). Ela é frequentemente utilizada para explorar o objeto de pesquisa que será analisado e é feita, basicamente, quando a teoria sobre o assunto a ser estudado é escassa e vaga. Seu foco não é explorar hipóteses pré-estabelecidas que sejam específicas à pesquisa.

A pesquisa descritiva é baseada na identificação, registro, mensuração e análise das características e fatores relacionados com a pesquisa. Esse método estabelece procedimentos formais para a coleta de dados e apresenta um caráter mais quantitativo. Esta pesquisa trabalhou com questionário (com perguntas objetivas e diretas), distribuído a três gestores e um proprietário.

Por se tratar de um estudo de caso que envolve dois campos gerenciais (finanças e marketing), foi utilizado o método de pesquisa mista, ou seja, que contenha tanto um caráter exploratório como descritivo. A abordagem metodológica adotada, portanto, foi uma pesquisa de campo qualitativa, descritiva e exploratória.

A definição dos procedimentos metodológicos, nos quais são apontados os caminhos e as técnicas seguidas para a investigação, são relevantes para a análise do complexo campo que é uma clínica especializada.

Em relação ao método: inicialmente estabeleceu-se:

1) Um protocolo de pesquisa. A formação de um quadro sinótico estruturado, com as perguntas realizadas e os objetivos a serem atingidos com as respostas do proprietário e principais gestores. De acordo com Sampieri, Collado e Lucio (2006) as entrevistas semiestruturadas "baseiam-se em um guia de assuntos e questões e o pesquisador tem liberdade de introduzir mais questões para a precisão de conceitos e obter mais informações sobre os temas pesquisados"; (PICCHIAl, 2012).

2) Uma pesquisa de campo. Aplicação da entrevista estruturada como meio de coleta de dados e informações, junto ao sócio proprietário e principais executivos. A entrevista é como uma forma de conversa assimétrica, em que uma das partes procura coletar dados e a outra se apresenta como fonte de informação (GIL, 2007). 
A pesquisa de campo caracterizou-se exploratória e descritiva. É exploratória, pois se pretendeu apresentar mais informações, proporcionando aos leitores submeter o seu conhecimento sobre o tema pesquisado. É descritiva porque se pretendeu apresentar características sobre a gestão da clínica estudada.

A fundamentação vivencial das funções gerenciais dos gestores e o proprietário da pequena empresa foram coletados por meio da observação das entrevistas estruturadas. Captaram-se quais suas percepções sobre as funções gerenciais e seus princípios de gestão. Os pesquisadores observaram a clínica durante nove meses. Também foi aplicado um questionário com questões abertas para manifestação dos dirigentes. (PICCHIAI, 2015)

3) Avaliação da literatura. Definição da amostra de artigos relacionados, leitura e fichamento, para o melhor entendimento conceitual e contribuição teórica ao tema estudado. Procurou-se focar em artigos e teses sobre a gestão de MPE's. A amostra é composta por artigos científicos publicados entre 1963 e 2015 em periódicos e anais de congresso.

A realização de entrevistas estruturadas com o proprietário e/os gestores executivos da pequena empresa selecionados focou aspectos relacionados à gestão: marketing e finanças. $O$ instrumento utilizado para levantamento dos dados e informações destes fatos e fenômenos de gestão foi um roteiro de entrevista semiestruturado, contendo 50 perguntas, das quais três questões são fechadas abordando com maior objetividade os temas pesquisados $\mathrm{e}$ realizadas ao final da entrevista.

Além disso, o instrumento foi construído segundo a literatura e validado por meio de pré-testes e ajustes necessários. Durante a condução da entrevista, o pesquisador esclareceu aos entrevistados, os conceitos abordados nas questões da pesquisa. Dessa maneira, foi possível detectar a percepção do proprietário sobre o que ele entende por gestão e as técnicas utilizadas em sua empresa, evitando-se erros e má compreensão dos conceitos. Também foi analisado a coerência, a consistência e o alinhamento dos conceitos de estratégia, estrutura e competências organizacionais aplicadas as MPE's, por meio da percepção de seu proprietário e gestores. (PICCHIAI, 2015).

O período de observação foi de setembro de 2014 a maio de 2015. Além das cinquenta perguntas, foram realizadas mais sete para descrição da empresa. Posteriormente, as respostas dos entrevistados foram convertidas, após a aplicação da entrevista em categorias para análise qualitativa, e também permitiu que alguns dados e informações pudessem ser tabulados e analisados estatisticamente.

O método de pesquisa por meio de roteiro de entrevista estruturada (BLOOM, REENEN, 2006), para não perder o foco do tema tratado, é composto de algumas perguntas diretas e objetivas sobre o tema, permitindo utilizar os instrumentos da estatística descritiva. 


\section{Apresentação e discussão dos resultados}

\subsection{Caracterização da clínica}

O Hospital e Clínica de Olhos Jundiaí possui 25 anos de funcionamento contando com atendimentos especializados em catarata, glaucoma, plástica estética, córnea (ceratone), estrabismo, retina e vítreo, lentes de contato e cirurgia de miopia a laser além de possuir o serviço de pronto atendimento e diferenciação de serviços para idosos. Seu corpo clínico é qualificado nos exames e procedimentos que realiza. A clínica possui instalações e equipamentos modernos, além de convênios com diferentes planos de saúde.

A infraestrutura da clínica oftalmológica localiza-se em um prédio com $550 \mathrm{~m} 2$ de área útil e pode ser caracterizada por: cinco consultórios; uma sala cirúrgica; um centro de diagnose por imagem; um equipamento Galilei (Placido- Dual Scheimpflug) para cirurgias refrativas e de catarata; estruturas para exames computadorizados e para adaptação de lentes de contato. Já seu volume de atendimento mensal segue em média de 1500 a 2000 atendimentos.

Os valores da clínica são: ética, transparência, acolhimento, credibilidade, inovação, qualidade, responsabilidade social, acessibilidade a tratamentos. Define-se como sua missão: "atuar no mercado da saúde suplementar brasileiro, por meio da promoção de assistência médica especializada com foco no acolhimento ao paciente e desenvolvimento de nossos profissionais". Por fim, sua visão é baseada no seguinte enunciado: "alcançar a posição de referência regional na oferta de serviços oftalmológicos de qualidade, sendo resolutivo nos tratamentos, desenvolvendo profissionais e conduzindo uma gestão ética e profissional, com pessoas, instituições e operadoras de saúde".

\subsection{Análises}

A partir da análise da clínica, pode-se observar, por meio das entrevistas e questionários, que os gestores analisam os aspectos financeiros e tomam as decisões na área apenas baseados no fluxo de caixa e no faturamento. Estes instrumentos de gestão financeira não são suficientes para uma análise mais apurada segundo a literatura pesquisada. Dessa maneira, não é possível perceber se o crescimento da empresa é rentável e sustentável. Têm-se limites de utilização de ferramentas específicas para as MPEs. Juntamente com as características cognitivas de seus gestores, com relação às suas formações profissionais.

A tendência de crescimento maior das despesas em relação com as receitas é originada do desalinhamento das áreas analisadas com a estratégia adotada de crescimento.

Quanto à área de marketing/comercialização, nota-se que apesar de oferecer um bom serviço, a clínica não consegue apresentar diferenciais perceptíveis do que fornece aos clientes. Tem existência de um plano de 
ESTUDO DE CASO DE UMA PEQUENA CLÍNICA:

FINANÇAS E MARKETING

marketing, como avaliação do mercado, concorrentes e público-alvo. Mas, foram evidenciadas muitas deficiências no plano operacional para sua execução. Identificou-se também, que há demanda para aumentar o número de cirurgias e, além disso, a estrutura e os equipamentos disponíveis não estão sendo utilizados em sua plena capacidade uma vez que, opta-se por focar em consultas que possuem menor valor agregado que as cirurgias, podendo realizar um melhor retorno financeiro.

As percepções da clientela, seja os pacientes ou mesmo os dirigentes de planos, necessitam ser bem analisadas, para assim, atingi-los da forma mais eficiente e eficaz. A satisfação é o resultado de uma transação de serviço único, enquanto que o julgamento sobre a qualidade de serviço se desenvolve após um período de numerosas transações de serviço (PARASURAMAN, 1988).

No contexto geral da empresa, observou-se que os entrevistados não declararam um plano de negócios estruturado, de tal modo que o funcionamento das áreas internas e a relação dessas com os seu ambiente externo pode ser prejudicada pela falta de alinhamento entre os membros e de uma definição clara dos objetivos pretendidos e de como atingi-los.

Nota-se que há certa dificuldade para a clínica em aplicar técnicas gerenciais no que se refere à utilização de conceitos de gestão e implementação desses conceitos na prática. Percebe-se a maior tangibilidade dos serviços da clínica e das MPEs no geral, uma vez que o serviço é mais personalizado e o paciente identifica uma relação de confiança com o médico. É evidente nesse contexto o aumento do custo variável das atividades desenvolvidas devido à personalização do serviço prestado.

A Tabela 1 e o Gráfico 1 mostram as receitas vindas das operadoras com que eles têm convênio, e há um queda nessas receitas. Já a Tabela 2 e o Gráfico 2 são receitas advindas das particulares e evidencia-se um aumento representativo destas. Além disso, a Tabela 3 e o Gráfico 3 demonstram as despesas totais da clínica e pode-se observar que elas estão crescendo mês a mês e, então, a Tabela 4 e o Gráfico 4 demonstram que as despesas estão superando as receitas e isso pode ser um fator relevante se permanecer nesse cenário. Dessa forma, a Tabela 5 e o Gráfico 5 mostram o decréscimo do resultado líquido obtido pela clínica em um ano, e a Tabela 6 dos demonstrativos financeiros organiza os tipos de receitas e despesas, assim como capital para investimento da clínica observada.

A Tabela 1 e o Gráfico 1 mostram as receitas, mês a mês da empresa, ao longo de treze meses. Pode-se observar que as receitas da clínica provenientes das operadoras apresentaram uma redução nas receitas nos meses de fevereiro e março em decorrência do cenário de recessão econômica que já era sentido em 2015, um dos possíveis impactos da crise econômica para a queda na receita das operadoras A crise impacta as receitas das operadoras na medida em que a população em geral apresenta uma menor cobertura de planos de saúde em momentos como esse. Isso se dá tanto nos planos individuais e familiares bem como na redução de cobertura decorrente da demissão de funcionários em diversos setores da economia. 
Tabela 1. Receitas das operadoras

\begin{tabular}{|c|c|c|c|}
\hline \multicolumn{4}{|c|}{ Receitas das operadoras } \\
\hline \multirow{3}{*}{ Ano } & Mês & Receita & $\%$ \\
\hline \multirow{4}{*}{} & Janeiro & $\mathrm{R} \$ 204.894,56$ & $* * *$ \\
\cline { 2 - 4 } & Fevereiro & $\mathrm{R} \$ 185.018,84$ & $-10 \%$ \\
\cline { 2 - 4 } & Março & $\mathrm{R} \$ 212.292,49$ & $\mathbf{1 5 \%}$ \\
\cline { 2 - 4 } & Abril & $\mathrm{R} \$ 179.994,41$ & $-15 \%$ \\
\cline { 2 - 4 } & Maio & $\mathrm{R} \$ 240.886,25$ & $\mathbf{3 4 \%}$ \\
\cline { 2 - 4 } & Junho & $\mathrm{R} \$ 242.290,68$ & $\mathbf{1 \%}$ \\
\cline { 2 - 4 } & Julho & $\mathrm{R} \$ 261.427,83$ & $\mathbf{8} \%$ \\
\cline { 2 - 4 } & Agosto & $\mathrm{R} \$ 203.783,74$ & $-\mathbf{2 2} \%$ \\
\cline { 2 - 4 } & Setembro & $\mathrm{R} \$ 284.944,93$ & $\mathbf{4 0 \%}$ \\
\cline { 2 - 4 } & Outubro & $\mathrm{R} \$ 288.259,70$ & $\mathbf{1 \%}$ \\
\cline { 2 - 4 } & Novembro & $\mathrm{R} \$ 251.411,01$ & $-13 \%$ \\
\cline { 2 - 4 } & Dezembro & $\mathrm{R} \$ 239.475,83$ & $-\mathbf{5} \%$ \\
\hline 2015 & Janeiro & $\mathrm{R} \$ 304.100,03$ & $\mathbf{2 7} \%$ \\
\hline
\end{tabular}

Gráfico 1. Receitas das operadoras

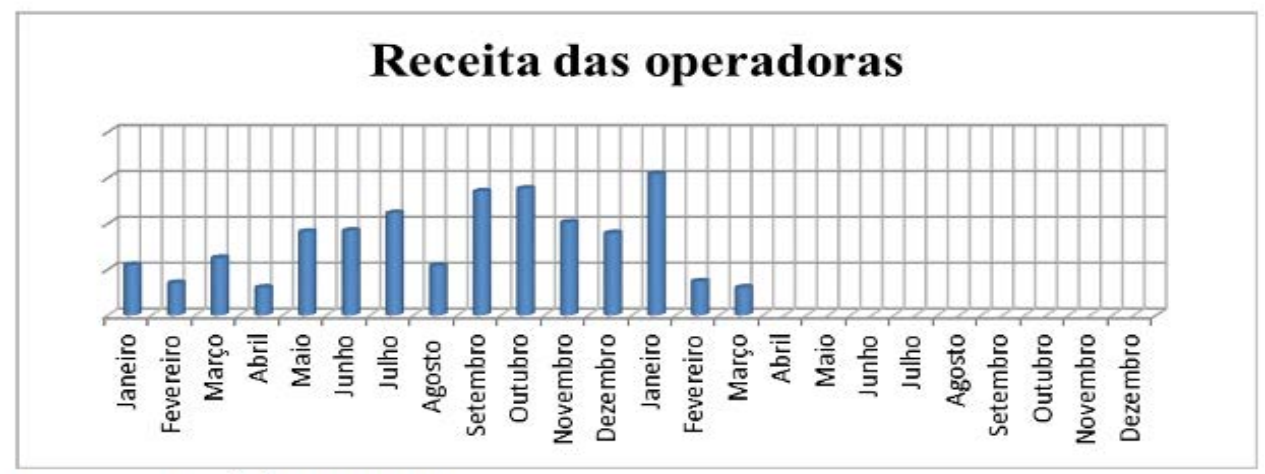

Fonte : Empresa HCJundiaí-2014 e 2015 
Tabela 2. Receita Particular

\begin{tabular}{|c|c|c|c|}
\hline \multicolumn{3}{|c|}{ Receita Particular } \\
\hline Ano & Mês & Receita & $\%$ \\
\hline \multirow{4}{*}{} & Janeiro & $\mathrm{R} \$ 154.903,55$ & $* * *$ \\
\cline { 2 - 4 } & Fevereiro & $\mathrm{R} \$ 138.298,35$ & $-\mathbf{1 1} \%$ \\
\cline { 2 - 4 } & Março & $\mathrm{R} \$ 155.459,00$ & $\mathbf{1 2 \%}$ \\
\cline { 2 - 4 } & Abril & $\mathrm{R} \$ 117.611,30$ & $-\mathbf{2 4 \%}$ \\
\cline { 2 - 4 } & Maio & $\mathrm{R} \$ 122.965,70$ & $\mathbf{5 \%}$ \\
\cline { 2 - 4 } & Junho & $\mathrm{R} \$ 122.315,80$ & $-\mathbf{- 1 \%}$ \\
\cline { 2 - 4 } & Julho & $\mathrm{R} \$ 127.303,90$ & $\mathbf{4} \%$ \\
\cline { 2 - 4 } & Agosto & $\mathrm{R} \$ 137.074,30$ & $\mathbf{8 \%}$ \\
\cline { 2 - 4 } & Setembro & $\mathrm{R} \$ 150.901,80$ & $\mathbf{1 0 \%}$ \\
\cline { 2 - 4 } & Outubro & $\mathrm{R} \$ 144.640,00$ & $-\mathbf{4} \%$ \\
\cline { 2 - 4 } & Novembro & $\mathrm{R} \$ 110.888,10$ & $-\mathbf{2 3} \%$ \\
\cline { 2 - 4 } & Dezembro & $\mathrm{R} \$ 100.112,80$ & $-\mathbf{1 0} \%$ \\
\hline 2015 & Janeiro & $\mathrm{R} \$ 138.724,00$ & $\mathbf{3 9} \%$ \\
\hline
\end{tabular}

Fonte: Empresa HCJundiaí-2014 e 2015

A Tabela 2 e o Gráfico 2 demonstram a distribuição das receitas dos particulares, com tendências ao crescimento. Conforme citado, o panorama econômico tem tornado difícil a prestação de serviços para clientes que utilizam convênios. Do ponto de vista das receitas particulares, há um esforço da clínica em atrair clientes particulares os quais estão aumentando nesse período. A tendência de crescimento dos últimos meses nessas receitas pode ser atribuída em um esforço da clínica tanto em investir no seu corpo clínico para aumentar a qualidade da prestação de seus serviços e também em um esforço em comunicar essa qualidade diferencial para os seus potenciais pacientes.

Gráfico 2. Receita Particular

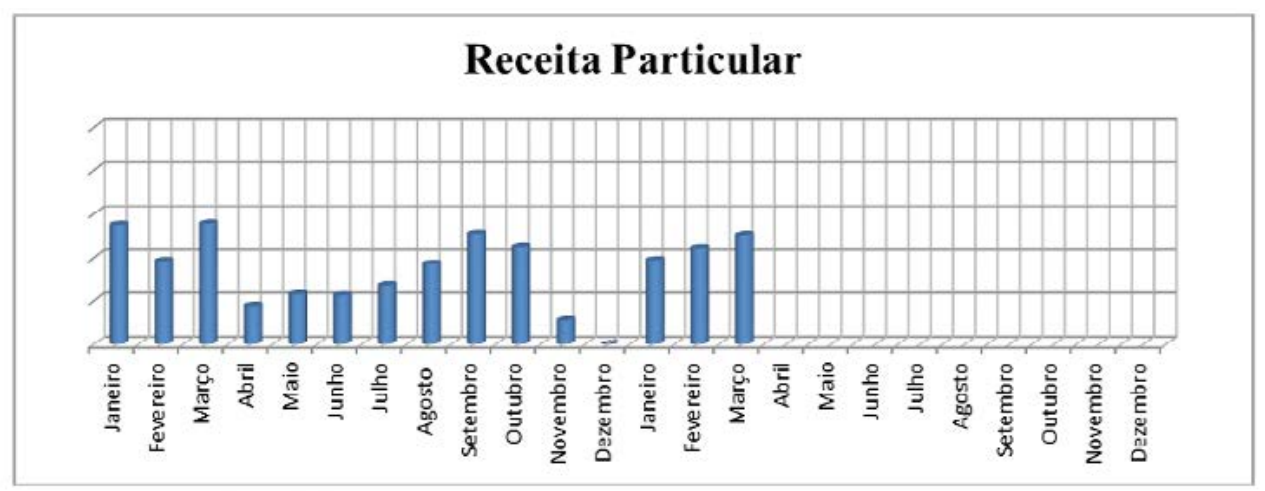

Fonte: Empresa HCJundiaí-2014 e 2015 


\begin{tabular}{|c|c|c|c|}
\hline \multicolumn{4}{|c|}{ Despesa Total } \\
\hline Ano & Mês & Despesa Total & $\%$ \\
\hline \multirow{4}{*}{} & Janeiro & $\mathrm{R} \$ 367.840,39$ & $* * *$ \\
\cline { 2 - 4 } & Fevereiro & $\mathrm{R} \$ 339.633,70$ & $-\mathbf{8} \%$ \\
\cline { 2 - 4 } & Março & $\mathrm{R} \$ 362.426,16$ & $\mathbf{7 \%}$ \\
\cline { 2 - 4 } & Abril & $\mathrm{R} \$ 409.135,88$ & $\mathbf{1 3 \%}$ \\
\cline { 2 - 4 } & Maio & $\mathrm{R} \$ 336.327,74$ & $\mathbf{- 1 8 \%}$ \\
\cline { 2 - 4 } & Junho & $\mathrm{R} \$ 372.880,17$ & $\mathbf{1 1 \%}$ \\
\cline { 2 - 4 } & Julho & $\mathrm{R} \$ 329.668,93$ & $\mathbf{- 1 2} \%$ \\
\cline { 2 - 4 } & Agosto & $\mathrm{R} \$ 342.022,29$ & $\mathbf{4 \%}$ \\
\cline { 2 - 4 } & Setembro & $\mathrm{R} \$ 354.488,44$ & $\mathbf{4 \%}$ \\
\cline { 2 - 4 } & Outubro & $\mathrm{R} \$ 414.037,74$ & $\mathbf{1 7 \%}$ \\
\cline { 2 - 4 } & Novembro & $\mathrm{R} \$ 412.040,97$ & $\mathbf{0 \%}$ \\
\cline { 2 - 4 } & Dezembro & $\mathrm{R} \$ 433.155,03$ & $\mathbf{5 \%}$ \\
\hline 2015 & Janeiro & $\mathrm{R} \$ 376.917,68$ & $\mathbf{- 1 3 \%}$ \\
\hline
\end{tabular}

Fonte: Empresa HCJundiaí-2014 e 2015

Gráfico 3:Despesa total

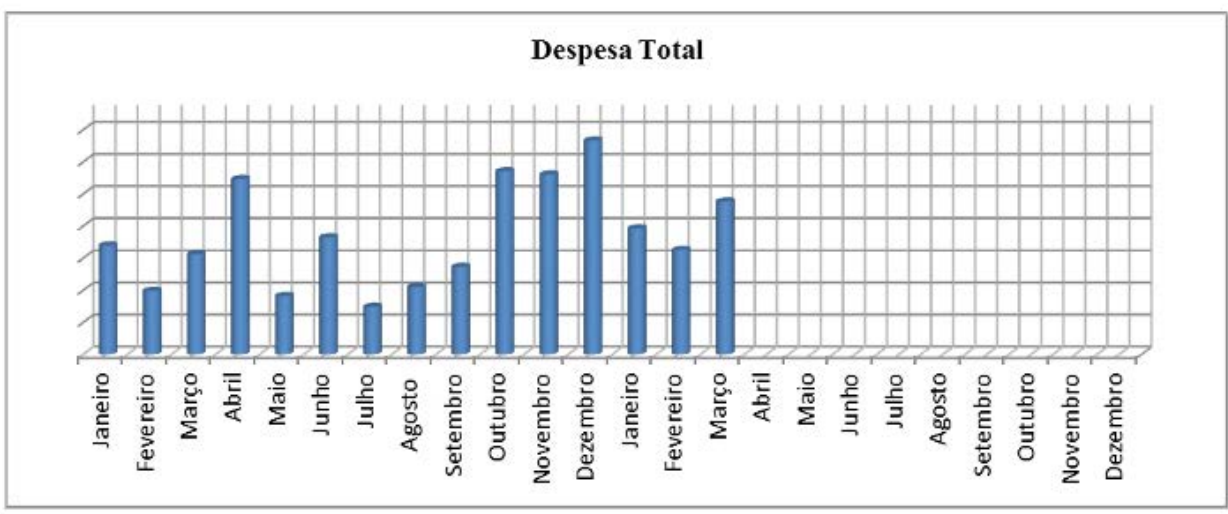

Fonte: Empresa HCJundiaí-2014 e 2015

A Tabela 3 e o Gráfico 3 demonstram as despesas totais da clínica que apresentaram altos níveis nos meses de outubro, novembro e dezembro de 2014 porém, registraram quedas nos meses seguintes. A elevação das despesas nos referidos meses pode ser atribuída tanto ao crescimento vegetativo dos salários dos funcionários bem como do aumento da folha de pagamentos devido à novas contratações a fim de aumentar a especialização dos serviços gerenciais. Vale ainda ressaltar que os preços dos materiais e medicamentos utilizados na clínica apresentam historicamente aumentos sempre superiores à inflação. 
ESTUDO DE CASO DE UMA PEQUENA CLÍNICA:

FINANÇAS E MARKETING

Tabela 4: Receita X despesa

\begin{tabular}{|l|l|l|l|}
\hline \multicolumn{3}{|l|}{ Receita X Despesa } \\
\hline Ano & Mês & Receitas Totais & Despesas Totais \\
\hline \multirow{8}{*}{} & Janeiro & $\mathrm{R} \$ 359.798,11$ & $\mathrm{R} \$ 367.840,39$ \\
\cline { 2 - 4 } & Fevereiro & $\mathrm{R} \$ 323.317,19$ & $\mathrm{R} \$ 339.633,70$ \\
\cline { 2 - 4 } & Março & $\mathrm{R} \$ 367.751,49$ & $\mathrm{R} \$ 362.426,16$ \\
\cline { 2 - 4 } & Abril & $\mathrm{R} \$ 447.605,71$ & $\mathrm{R} \$ 409.135,88$ \\
\cline { 2 - 4 } & Maio & $\mathrm{R} \$ 363.851,95$ & $\mathrm{R} \$ 336.327,74$ \\
\cline { 2 - 4 } & Junho & $\mathrm{R} \$ 364.606,48$ & $\mathrm{R} \$ 372.880,17$ \\
\cline { 2 - 4 } & Julho & $\mathrm{R} \$ 388.731,73$ & $\mathrm{R} \$ 329.668,93$ \\
\cline { 2 - 4 } & Agosto & $\mathrm{R} \$ 340.858,04$ & $\mathrm{R} \$ 342.022,29$ \\
\cline { 2 - 4 } & Setembro & $\mathrm{R} \$ 435.846,73$ & $\mathrm{R} \$ 354.488,44$ \\
\cline { 2 - 4 } & Outubro & $\mathrm{R} \$ 432.899,70$ & $\mathrm{R} \$ 414.037,74$ \\
\cline { 2 - 4 } & Novembro & $\mathrm{R} \$ 362.299,11$ & $\mathrm{R} \$ 412.040,97$ \\
\cline { 2 - 4 } & Dezembro & $\mathrm{R} \$ 339.588,63$ & $\mathrm{R} \$ 433.155,03$ \\
\hline \multirow{2}{*}{2015} & Janeiro & $\mathrm{R} \$ 442.824,03$ & $\mathrm{R} \$ 376.917,68$ \\
\hline
\end{tabular}

Fonte: Empresa HCJundiá-2014 e 2015

Gráfico 4: Receita X despesa

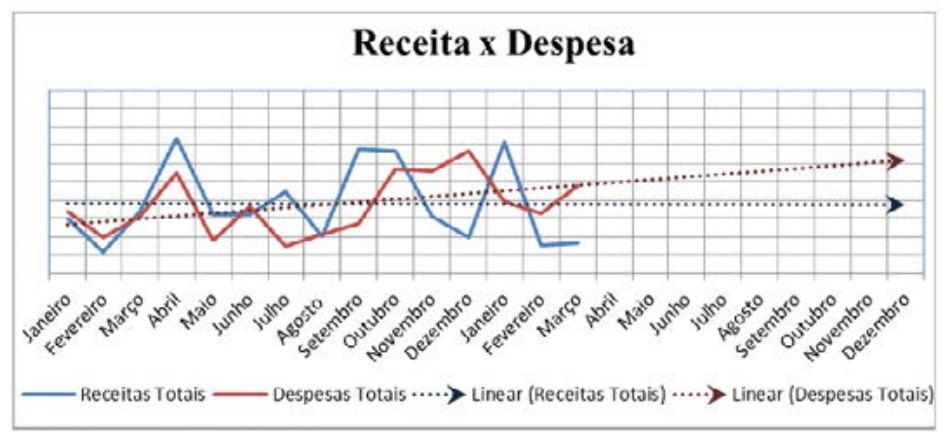

Fonte: Empresa HCJundiaí-2014

A Tabela 4 e o Gráfico 4 demonstram as tendências das receitas e despesas totais, com tendência crescente maior nas despesas se comparadas às receitas obtidas. Essa diferença considerada negativa entre os resultados pode ser atribuída especialmente à redução das receitas de pacientes com convênios e ao aumento das despesas com a folha de pagamentos. 
Tabela 5: Resultado líquido

\begin{tabular}{|c|c|c|c|}
\hline \multicolumn{3}{|c|}{ Resultado Líquido } \\
\hline Ano & Mês & Resultado & $\%$ \\
\hline \multirow{4}{*}{} & Janeiro & $-\mathrm{R} \$ 8.042,28$ & $* * *$ \\
\cline { 2 - 4 } & Fevereiro & $-\mathrm{R} \$ 16.316,51$ & $-103 \%$ \\
\cline { 2 - 4 } & Março & $\mathrm{R} \$ 5.325,33$ & $\mathbf{1 3 3 \%}$ \\
\cline { 2 - 4 } & Abril & $\mathrm{R} \$ 38.469,83$ & $\mathbf{6 2 2} \%$ \\
\cline { 2 - 4 } & Maio & $\mathrm{R} \$ 27.524,21$ & $-\mathbf{2 8 \%}$ \\
\cline { 2 - 4 } & Junho & $-\mathrm{R} \$ 8.273,69$ & $-130 \%$ \\
\cline { 2 - 4 } & Julho & $\mathrm{R} \$ 59.062,80$ & $\mathbf{8 1 4 \%}$ \\
\cline { 2 - 4 } & Agosto & $-\mathrm{R} \$ 1.164,25$ & $-102 \%$ \\
\cline { 2 - 4 } & Setembro & $\mathrm{R} \$ 81.358,29$ & $\mathbf{7 0 8 8} \%$ \\
\cline { 2 - 4 } & Outubro & $\mathrm{R} \$ 18.861,96$ & $-\mathbf{7 7} \%$ \\
\cline { 2 - 4 } & Novembro & $-\mathrm{R} \$ 49.741,86$ & $-\mathbf{3 6 4} \%$ \\
\cline { 2 - 4 } & Dezembro & $-\mathrm{R} \$ 93.566,40$ & $-\mathbf{8 8} \%$ \\
\hline 2015 & Janeiro & $\mathrm{R} \$ 65.906,35$ & $\mathbf{1 7 0} \%$ \\
\hline
\end{tabular}

Fonte: Empresa HCJundiaí-2014 e 2015

Gráfico 5: Resultado líquido

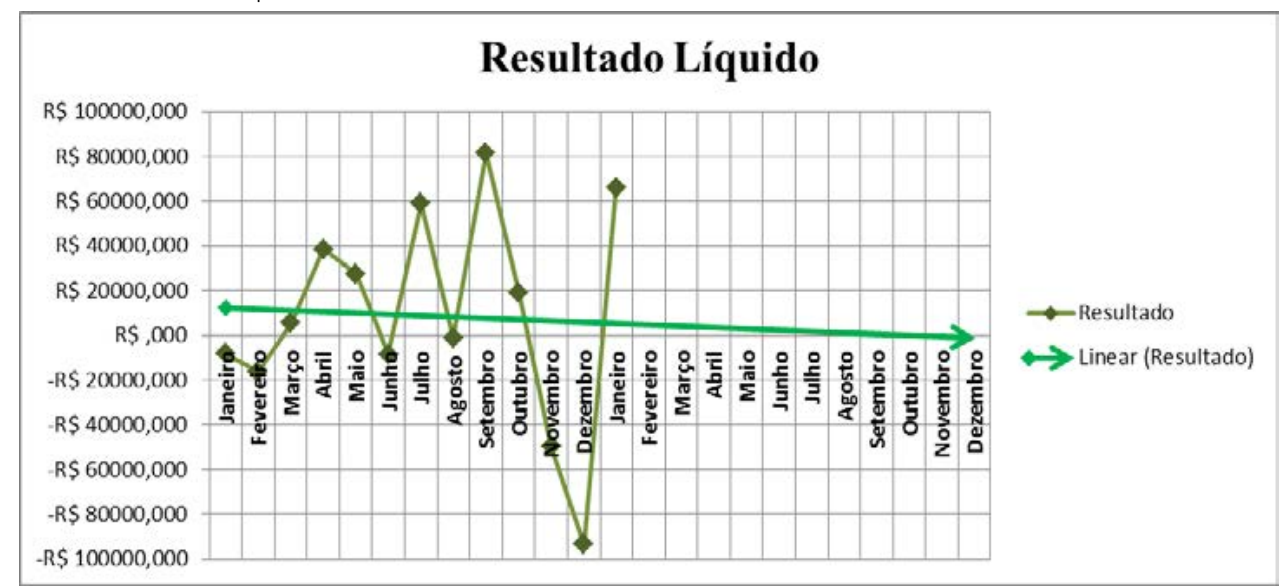

Fonte: Empresa HCJundiaí-2014

A Tabela 5 e o Gráfico 5 demonstram a tendência de queda no resultado líquido da clínica. Isso é destacado como um mero reflexo do que foi indicado pelas crescentes despesas principalmente com pessoal e das quedas na receita provenientes das operadoras. 
ESTUDO DE CASO DE UMA PEQUENA CLÍNICA:

FINANÇAS E MARKETING

Tabela 6: Resultados financeiros

\begin{tabular}{|c|c|}
\hline Médias Mensais & $\mathbf{2 0 1 4}$ \\
\hline Rec. Operadora & $\mathrm{R} \$ \quad 232.890,02$ \\
\hline Rec. Particular & $\mathrm{R} \$ \quad 131.872,88$ \\
\hline Receita Total & $\mathrm{R} \$ \quad 377.262,91$ \\
\hline Despesa Total & $\mathrm{R} \$ \quad 372.804,79$ \\
\hline Resultado Líquido & $\mathrm{R} \$ 4.458,12$ \\
\hline Total Anual & $\mathbf{2 0 1 4}$ \\
\hline Faturamento & $\mathrm{R} \$ 4.527 .154,87$ \\
\hline Despesas & $\mathrm{R} \$ 4.473 .657,44$ \\
\hline Resultado Líquido & $\mathrm{R} \$ 53.497,43$ \\
\hline Fonte: Empresa HCJundiaí-2014
\end{tabular}

Fonte: Empresa HCJundiaí-2014

A Tabela 6 mostra as médias mensais dos resultados da clínica em 2014 e os valores totais para o ano de 2014 que refletem as tendências de variação observadas anteriormente.

$\mathrm{Na}$ Tabela 7, é interessante observar que os resultados agregados do ano de 2014 e 2015 não refletem o cenário de queda destacados anteriormente, uma vez que os demonstrativos para os meses de fevereiro e março de 2015, nos quais se observou uma redução considerável nas receitas das operadoras, não haviam ainda sido fornecidos para a análise.

Tabela 7: Demonstrativo de resultado - DRE

\begin{tabular}{|c|c|c|c|c|}
\hline \multicolumn{5}{|l|}{ Demonstrativo de Resultado - DRE } \\
\hline Regime de Caixa & 2014 & \multirow[b]{2}{*}{ Novembro } & \multirow[b]{2}{*}{ Dezembro } & 2015 \\
\hline & Outubro & & & Janeiro \\
\hline (=) Receita Operacional Bruta & $R \$ 432.899,70$ & $\mathrm{R} \$ 362.299,11$ & $R \$ 339.588,63$ & $R \$ \quad 442.824,03$ \\
\hline (+) Receita de Operadoras & $\mathrm{R} \$ 288.259,70$ & $\mathrm{R} \$ 251.411,01$ & $\mathrm{R} \$ 239.475,83$ & $\mathrm{R} \$ 304.100,03$ \\
\hline (+) Receita de Particular & $\mathrm{R} \$ 144.640,00$ & $\mathrm{R} \$ 110.888,10$ & $\mathrm{R} \$ 100.112,80$ & R\$ $138.724,00$ \\
\hline (-) Impostos sobre Serviços & $9.601,70$ & $\mathrm{R} \$ \quad 10.514,34$ & $6.485,11$ & $6.295,55$ \\
\hline (=) Receita Operacional Líquida & $\mathrm{R} \$ 423.298,00$ & $\mathrm{R} \$ 351.784,77$ & $\mathrm{R} \$ 333.103,52$ & $\mathrm{R} \$ \quad 436.528,48$ \\
\hline (-) Custo dos Serviços Prestados & $\mathrm{R} \$ \quad 143.045,92$ & $\mathrm{R} \$ \quad 121.750,21$ & $\mathrm{R} \$ \quad 27.042,94$ & $\mathrm{R} \$ \quad 25.579,57$ \\
\hline (=) Lucro Bruto & $\mathrm{R} \$ 280.252,08$ & $\mathrm{R} \$ 230.034,56$ & $\mathrm{R} \$ 306.060,58$ & $\mathrm{R} \$ \quad 410.948,91$ \\
\hline (-) Despesas Administrativas & $\mathrm{R} \$ \quad 168.942,24$ & $\mathrm{R} \$ \quad 181.187,25$ & $\mathrm{R} \$ 306.587,55$ & $\mathrm{R} \$ 251.666,30$ \\
\hline (=) Lucro antes do IR & $\mathrm{R} \$ \quad 111.309,84$ & $\mathrm{R} \$ \quad 48.847,31$ & 526,97 & $R \$ 159.282,61$ \\
\hline (-) Imposto de Renda & $16.644,29$ & $\mathrm{R} \$ \quad 21.109,73$ & $\mathrm{R} \$ \quad 22.431,51$ & $\mathrm{R} \$ \quad 17.543,70$ \\
\hline (-) Contribuição Social & $5.665,59$ & $7.341,44$ & 469,92 & $5.694,56$ \\
\hline (=) Lucro Líquido & $88.999,96$ & $\mathrm{R} \$ \quad 20.396,14$ & $R \$ \quad 23.428,40$ & $\mathrm{R} \$ 136.044,35$ \\
\hline (-) Pró-labóre & $40.000,00$ & $\mathrm{R} \$ \quad 40.000,00$ & $\mathrm{R} \$ \quad 40.000,00$ & $\mathrm{R} \$ \quad 40.000,00$ \\
\hline$(-)$ Outras retiradas & $23.333,00$ & $R \$ \quad 23.333,00$ & $R \$ \quad 23.333,00$ & $\mathrm{R} \$ \quad 23.333,00$ \\
\hline (=) Fluxo de Caixa Livre para a Empresa & $25.666,96$ & $-R \$ \quad 42.936,86$ & $-R \$ \quad 86.761,40$ & $\mathrm{R} \$ \quad 72.711,35$ \\
\hline (-) Investimentos & $6.805,00$ & $6.805,00$ & $6.805,00$ & $6.805,00$ \\
\hline (=) Fluxo de Caixa Livre para os Sócios & $18.861,96$ & -R\$ $49.741,86$ & $-R \$ \quad 93.566,40$ & $\mathrm{R} \$ \quad 65.906,35$ \\
\hline ÍNDICES & & & & \\
\hline Lucratividade & 0,21 & 0,06 & 0,07 & 0,31 \\
\hline
\end{tabular}

Fonte: Empresa HCJundiaí- 2014 e 2015 
Por meio de entrevistas com os gestores da clínica e com a responsável pela área de marketing, pode-se observar tanto o discurso quanto às práticas da clínica. Um dos pontos destacados pela empresa é o seu foco no atendimento resolutivo devido à listagem ampla de serviços prestados de forma humanizada.

Quanto à comercialização desses serviços, pode-se observar que a clientela está dispersa uma vez que a carteira de operadores é pulverizada. Isso pode ser uma fonte de poder para a empresa que não fica dependente apenas de uma ou poucas operadoras.

A responsável pelo marketing acompanha cada uma das reclamações dos clientes do serviço de atendimento ao cliente da clínica. Isso faz parte do pós-venda da clínica uma vez que sua estratégia de marketing está mais voltada em destacar a qualidade do seu corpo clínico como um diferencial frente aos seus concorrentes.

Referente ao aumento na tangibilidade dos serviços, utiliza-se a especialização dos médicos e a melhora da tecnologia empregada nos serviços, e também à segmentação do espaço da clínica de acordo com a complexidade e a gravidade dos casos, além da separação física dos atendimentos de clientes particulares e de convênios.

Finalmente, deve-se ressaltar que a clínica não conta com metas de volume de atendimento definidas. Isso pode ser um problema para comparar diferenças entre o que era pretendido e o que foi realizado.

\section{Considerações finais}

A intuição, a vivência e a experiência empresarial do proprietário foram consideradas relevantes nas tomadas de decisões e sobrevivência da clínica. Por outro lado muitas das ferramentas gerenciais não são utilizadas, apesar do ambiente organizacional assim exigir.

Conforme identificado, a reputação do corpo clínico foi caracterizada como muito importante para a imagem da clínica frente aos seus clientes e apresentando-se como um diferencial em relação aos concorrentes. A sustentabilidade da clínica nos últimos 20 anos aponta para resultados financeiros e comerciais satisfatórios com margens de rentabilidade menores.

\section{Referências}

BARNEY, Jay B. Administração estratégica e vantagem competitiva: conceitos e casos. 3. ed. São Paulo: Pearson Prentice-Hall, 2011.

BERTERO, Carlos O; CALDAS, Miguel P.;WOOD JR,Thomas. Produção Científica em Administração de Empresas: Produção Científica em Administração de Empresas: Provocações, Insinuações e Contribuições para um Debate Local. RAC. Revista de Administração Contemporânea, São Paulo / Brasil, v. 3, n. 1, p.147178, Jan/Abr, 2001. 
ESTUDO DE CASO DE UMA PEQUENA CLÍNICA:

FINANÇAS E MARKETING

BLOCHING, Björn; STOCK, Harald F.; SCHEEL, Jochen. Journal of Medical Market. Vol. 25 Tópico 6, p747-772. Alemanha: 2008.

BLOOM, Nick, REENEM, Jhon Vem. Measuring and Explaining Management Practices Across Firms and countries. Center of Economies Performance - CEP Discussions Paper $n^{\circ}$ 716, London school, 2006.

BULGACOV, Sergio et. al. Administração Estratégica: teoria e prática. São Paulo: Atlas, 2007.

CHANDLER, D. Jr. Alfred. Strategy and sctruture; chapters in the history of the industrial enterprise. Cambridge, MA: 1962.

DAVENPORT, T.; HARRIS, J. G. Competição analítica; vencendo através de uma nova ciência. Rio de Janeiro: Campus, 2007.

DUTRA, Joel Souza. Competências: conceitos e instrumentos para a gestão de pessoas na empresa moderna. São Paulo: Atlas, 2004.

FAGUNDES, Fábio Mello; GIMENEZ, Fernando Antonio Prado. Ambiente, estratégica e desempenho em micro e pequenas empresas. São Paulo, Encontro XXXIII ANPAD, 19 a 23 de Setembro de 2009.

FERNANDES, Bruno Henrique Rocha. Gestão estratégica de pessoas com foco em competências. Rio de Janeiro: Campus/Elsevier, 2013.

FREEMAN, R. E.; MCVEA, J. A stakeholder approach to strategic management. In: HITT, M.; FREEMAN, E.; HARRISON, J. Handbook of strategic management. Oxford: Blackwell Publishing, 2000.

GIL, Antonio Carlos. Como elaborar projetos de pesquisa. 4. ed. São Paulo: Atlas, 2007.

GLASCOFF, D. W. Evaluation of the Consumer Model: Relationship Between Patients' Expectations, Perceptions, and Satisfaction with Care. Marketing Health Services, v. 22, p. 44. Chicago Spring, 2002.

GONÇALVES, Shirley Rodrigues; SOUZA, Vanessa Cristina; SILVIA, Vanessa Aparecida; SILVA, Welton Ramos Vieira. Investimentos em recursos humanos em dez micro e pequenas empresas de Osasco e região. Revista Científica Hermes 3: p. 14-23, 2010.

KAPLAN, Robert. S; NORTON, David P. A estratégia em Ação: balanced scorecard. Rio de Janeiro: Editora Campus. 2001.

KOTLER, Philip. Princípios de marketing. São Paulo: Prentice Hall, 2000.

LEONE, Nilda Maria de C. P. Guerra. As especificidades das pequenas e médias empresas. Revista de Administração, São Paulo, v.34, n.2, p. 91-94, Abr./Jun. 1999.

LEVINSON, J. C. Marketing de Guerrilha: Táticas e armas para obter grandes lucros com pequenas e medias empresas. 1.ed. São Paulo: Best Seller, 1989.

MARCONI, M. A.; LAKATOS, E. M. Fundamentos da metodologia científica. $4^{\mathrm{a}}$ ed. Ver. Ampl. São Paulo: Atlas, 2001.

MARTINS, Paulo Sérgio; FILHO, Edmundo Escrivão. O meio ambiente no contexto organizacional: uma reflexão sobre a dimensão ambiental em pequenas e médias empresas. VII Simpósio de Excelência em Gestão e Tecnologia, 2010.

MINTZBERG, H.; QUINN, J. B. O processo da estratégia. $3^{a}$ edição. Porto Alegre: Bookman, 2001.

PARASURAMAN, A., ZEITHAML, V.A; BERRY,L. SERVIQUAL: A multiple item scale for mensuring consumers percepcions of service quality. Jounal of Retailing. 1988, p. 35-48.

PICCHIAl, Djair. A Gestão de micro e pequenas empresas na percepção de seus 
proprietários e gestores: estratégias, estruturas e competências organizacionais. FAPESP. São Paulo 2012.

PICCHIAI, Djair. Estudo de caso de uma pequena clínica oftalmológica na cidade de Jundiaí. Relatório de pesquisa. EAESP - FGV e FACCAMP, Campo Limpo Paulista 2015.

PORTER, M. E. Competitive strategy: techniques for analysing industries and competitors. New York: Free Press, 1980.

PORTER, M. E; TEISBERG, E. O. Repensando a saúde: estratégias para melhorar a qualidade e reduzir os custos. Porto Alegre: Bookman, 2007.

SAMPIERI, Roberto H.; COLLADO, Carlos F.; LUCIO, Pilar B. Metodologia de Pesquisa. 3. Ed, São Paulo: McGraw Hill, 2006.

SEBRAE. Fatores condicionantes e taxa de mortalidade de empresas no Brasil. Disponível em: <http://www.sebrae.com.br/br/mortalidade_empresas/ resumoexecutivo.asp.> Acesso em: 15 fev. 2011. Sebrae, 2008.

SEBRAE_SP. Pesquisa: CausaMortis: o sucesso e o fracasso das empresas nos primeiros cinco anos de vida, 2013. Disponível em: < http://www.sebraesp. com.br/arquivos_site/bibliot eca/EstudosPesquisas/mpes_numeros/book\%20 de\%20pesquisas_mpes\%20paulistas_2015_web_v3.pdfwww.sebrae.com.br.> Acesso em: 24/03/2015.

TARN, David D. C. Journal of Medical Marketing. Vol. 8, Tópico 4, p303-310. 2008.

TURRINI, R. N. T. Percepção dos usuários sobre a resolutividade e a satisfação pelos serviços de saúde na região sudoeste da Grande São Paulo. Tese de Doutorado em Saúde Pública. Universidade de São Paulo. São Paulo, 2001.

VERGARA, Sylvia Constant. Projetos e relatórios de pesquisa em administração. 9 ed. São Paulo: Atlas, 2007.

YIN, R.K. Estudo de caso: planejamento e métodos. $3^{\text {a }}$ edição. Porto Alegre: Bookman, 2005. 